
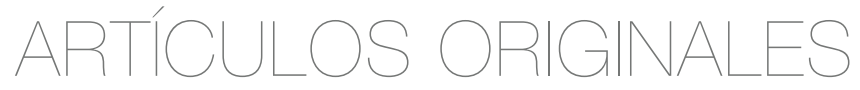

\title{
Emprendimiento académico y spin-off universitario: una revisión sistemática de la literatura
}

\author{
BEATRIZ M. TERÁN-PÉREZ* \\ CUITLÁHUAC VALDEZ LAFARGA** \\ ALEJANDRA MIRANDA FÉLIX***
}

* Doctoranda en Ciencias Administrativas. Universidad Autónoma de Occidente, Sinaloa, México. E-mail: bemaita13@gmail.com. ORCID: 0000-0002-7561-9938. Google Scholar: https://scholar.google.com/ citations?user=vdqizzgAAAAJ\&hl=es.

** Doctor en Ciencias. Universidad Autónoma de Occidente, Sinaloa, México. E-mail: cuitlahuac.valdez@udo.mx. ORCID: 0000-0001-7876-1430. Google Scholar: https://scholar.google.es/citations?user=6VTU-UgAAAA\&hl=es.

*** Doctora en Ciencias Administrativas. Universidad Autónoma de Sinaloa, Sinaloa, México. E-mail: amiranda@uas.edu.mx. ORCID: 0000-0001-5469-0834. Google Scholar: https://scholar.google.com/citations?user=1AGwtagAAAJ\&hl=en. 


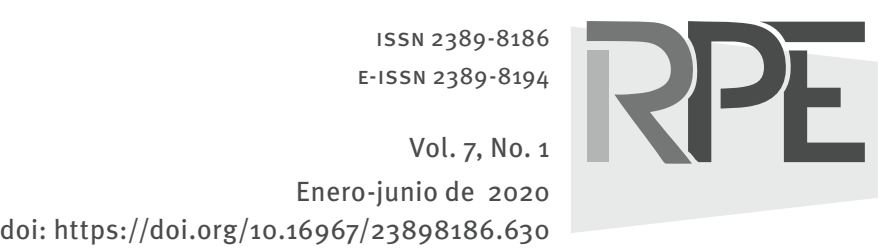

COMO CITAR ESTE ARTÍCULO

How to cite this article:

Terán-Pérez, B., Valdez, C. y Miranda, A. (2020). Emprendimiento académico y spin-off universitario: una revisión sistemática de la literatura. Revista Perspectiva Empresarial, 7(1), 87-103.

Recibido: 21 de octubre de 2019 Aprobado: 22 de enero de 2020
RESUMEN El presente estudio explora la literatura científica relacionada con el emprendimientoacadémicoyspin-offen elcontextouniversitarioconelfindeidentificarcómo está organizada la información en este campo de estudio, cuáles son los enfoques teóricos y metodológicos abordados en la literatura, así como detectar brechas para futuras líneas de investigación. Se realizó una revisión sistemática a 57 artículos indexados, obtenidos de la base de datos Scopus y publicados entre enero de 2014 y octubre de 2019. Primero se llevó a cabo un análisis bibliométrico a través del software VOSviewer, posteriormente se analizó el contenido del conjunto de estudios para cumplir los objetivos. Los resultados confirman que existe una creciente literatura relacionada al emprendimiento académico, pero fragmentada bajo enfoques teóricos heterogéneos. En suma, las metodologías cuantitativas van en ascenso y futuras investigaciones holísticas son sugeridas.

PALABRAS CLAVE emprendimiento académico, transferencia tecnológica, spin-off universitario y académico, universidad.

\section{Academic entrepreneurship and university spin-offs: A systematic literature review}

ABSTRACT This paper explores scientific literature related to academic entrepreneurship and spin-off in university settings with the aim of identifying how information in this study field is organized, which the theoretical and methodological approaches in the literature are, as well as detecting gaps for future research lines. A systematic review of 57 indexed papers was conducted, obtained from the Scopus database and published between January 2014 and October 2019. Firstly, a bibliometric analysis through the VOSviewer software was carried out, and subsequently, the content of the set of studies was analyzed to meet the aims of the research. The results confirm that there is growing literature related to academic entrepreneurship, but it is fragmented under heterogeneous theoretical approaches. In sum, quantitative methodologies are on the rise and future holistic research is suggested.

KEY WORDS academic entrepreneurship, technological transfer, university and academic spin-off, university. 


\section{Empreendedorismo acadêmico e spin-offs universitários: uma revisão sistemática da literatura}

RESUMO 0 presente estudo explora a literatura científica relacionada ao empreendedorismo acadêmico e à spin-offs no contexto universitário, a fim de identificar como as informações são organizadas nesse campo de estudo, quais são as abordagens teóricas e metodológicas abordadas na literatura, bem como detectar brechas para futuras linhas de pesquisa. Foi realizada uma revisão sistemática de 57 artigos indexados, obtidos na base de dados Scopus e publicados entre janeiro de 2014 e outubro de 2019. Uma análise bibliométrica foi realizada primeiro com o software VOSviewer e, em seguida, o conteúdo do conjunto de estudos foi analisado para atender aos objetivos. Os resultados confirmam que há uma crescente literatura relacionada ao empreendedorismo acadêmico, mas fragmentada sob abordagens teóricas heterogêneas. Em suma, metodologias quantitativas estão em ascensão e futuras pesquisas holísticas são sugeridas.

PALAVRAS-CHAVE empreendedorismo acadêmico, transferência de tecnologia, cisão universitária e acadêmica, universidade. 


\section{Introducción}

En una sociedad basada en el conocimiento, la universidad es un agente transformador esencial para el adecuado funcionamiento de los ecosistemas de emprendimiento y el sistema nacional de innovación puesto que conforma un vínculo que facilita la evolución del conocimiento que genera valor social o económico al llevarlo al mercado a través de alguna iniciativa emprendedora (Audretsch, 2014; Autio et al., 2014; Mosey, Guerrero and Greenman, 2016; Siegel, 2018). De igual manera las universidades son consideradas catalizadores para el desarrollo económico y social regional dado que son incubadoras naturales que crean nuevas ideas y tecnologías, promueven la creación de nuevos negocios y ofrecen una variedad de recursos y capacidades que contribuyen a crear ventajas competitivas (Etzkowitz et al., 2000; O'Shea, Chugh and Allen, 2008; Guerrero, Cunningham and Urbano, 2015).

De acuerdo con Bradley, Hayter y Link (2013) en las últimas décadas las universidades han ampliado sus actividades de emprendimiento debido especialmente a la colaboración con el sector productivo por medio de innovaciones tecnológicas que impactan el crecimiento económico regional. A la vez señalan la importancia de la cultura emprendedora universitaria que valora la transferencia de tecnología y apoya la comercialización de las investigaciones a través de patentes, licenciamientos y creación de spin-off.

A pesar de las expectativas y la atención significativa del papel de las universidades en la creación de spin-off generados a partir de investigaciones universitarias, los resultados en muchos contextos parecen desalentadores (Rasmussen, Mosey and Wright, 2015; Rao and Mulloth, 2017). El transitar de la universidad tradicional hacia una universidad emprendedora donde se caracterice por crear entornos en los que el emprendimiento basado en el conocimiento sea una fuerza motriz para llevar a cabo sus misiones (docencia, investigación y transferencia del conocimiento) implica cambios en términos de organización, cultura y estrategia (Etzkowitz et al., 2000; Guerrero and Urbano, 2012; O'Shea et al., 2014; Guerrero, Cunningham and Urbano, 2015).
Por lo anterior, diversos estudios exploran el por qué algunas universidades tienen más éxito que otras en la creación de empresas con base tecnológica; específicamente el cómo diferentes factores en el contexto universitario conducen al establecimiento y posterior desempeño de empresas basadas en la ciencia (Rasmussen, Mosey and Wright, 2015; Lockett and Wright, 2005).

Otro debate en la frontera del conocimiento está orientado a comprender cómo los diferentes agentes del ecosistema de emprendimiento - del cual la universidad es parte- operan, colaboran, toman decisiones, identifican beneficios 0 transforman sus roles y el papel que desempeñan esos agentes en el éxito de las empresas emergentes en el ámbito académico (Cunningham and Link, 2015; Boh, De-Haan and Strom, 2016; Hayter et al., 2018; Wright, Siegel and Mustar, 2017).

En ese mismo orden de ideas, según la clasificación de Carayannis (2017), Cunningham y O’Reilly (2018), en la literatura se han desarrollado líneas de investigación desde diferentes ámbitos/ niveles: macro; meso y micro. Bajo el enfoque macro se encuentran estudios relacionados con la incidencia del marco legal, económico y social en el emprendimiento académico y del impacto de este fenómeno en la región (Audretsch, 2014; Etzkowitz, 2017). En lo que respecta a estudios con enfoque meso, la literatura presenta estudios relacionados con procedimientos y prácticas de gestión organizacional para el fortalecimiento del ecosistema de emprendimiento universitario que permitan comercializar los resultados de investigación (Guerrero, Urbano and Gajón, 2017; Hayter et al., 2018). Finalmente las líneas de investigación con perspectiva micro estudian las características individuales del emprendedor o por grupos de investigación, la orientación emprendedora de las facultades, el cómo los emprendedores académicos identifican las oportunidades de negocios y cuál es la actitud hacia el emprendimiento y la intención de emprender de la comunidad universitaria (académicos, estudiantes y personal administrativo) (Clarysse, Tartari and Salter, 2011; Guerrero and Urbano, 2012; Urban and Chantson, 2017).

De acuerdo a las proposiciones anteriores se visualiza que el tópico de emprendimiento 
académico es multidisciplinario y los diferentes niveles en los que puede ser estudiado (macro, meso y micro) están relacionados. Específicamente el objetivo de explorar la literatura científica de frontera en emprendimiento académico y spinoff en el contexto universitario para identificar cómo está organizada la información en este campo de estudio, cuáles son los enfoques teóricos y metodológicos abordados en la literatura, así como detectar brechas para futuras líneas de investigación. Para ello se realiza una revisión sistemática de la literatura a partir de un protocolo riguroso de pasos para ejecutar la investigación y el análisis de la información que permita comprender lo que se ha hecho en el área de estudio y proporcione así una guía para desarrollar un marco sistemático y holístico para el tema (Schmitz et al., 2016; Mascarenhas et al., 2017).

La siguiente sección describe la metodología mediante la cual se realizó la investigación. Luego se presentan los principales resultados del análisis bibliométrico; se incluye la publicación por años, las áreas temáticas de investigación, publicaciones por revistas; $y$ a través del software VOSviewer se realizan mapas de citación, palabras clave y cocitación de autores. Posteriormente son presentados los principales resultados del análisis de contenido, los enfoques teóricos y metodológicos encontrados en los artículos revisados y las brechas para futuras líneas de investigación. Finalmente se presentan las conclusiones de la revisión de la literatura de frontera.

\section{Metodología}

Para evitar sesgos comunes en las revisiones de literatura tradicionales se utilizó un enfoque sistemático para proporcionar un resumen exhaustivo de la literatura relevante. Las revisiones sistemáticas mejoran la calidad del proceso de revisión y los resultados al emplear un procedimiento transparente y reproducible, el cual ha sido utilizado en las ciencias sociales (Liñán and Fayolle, 2015; Schmitz et al., 2016; Mascarenhas et al., 2017). En este estudio, el proceso comprende los siguientes pasos:
Búsqueda y descarga de literatura: se realizó una búsqueda dentro de la base de datos Scopus, el 23 de octubre de 2019; seleccionada por ser una de las más reconocidas debido a que provee publicaciones científicas de revistas, libros y actas de congresos, revisadas por pares en diversos campos de la ciencia y la tecnología.

Esa búsqueda consistió en utilizar los términos clave: "academic entrepreneurship"; "academic spin-off" y "university spin-off", dentro del portal de la base de datos seleccionada. Se utilizaron comillas para garantizar los resultados, operadores lógicos AND, OR y se condicionó la coincidencia en título, resumen y palabras clave de las publicaciones. Posteriormente se filtró la búsqueda de los documentos por fecha de publicación, no mayores a 6 años (de 2014 en adelante); lo anterior, con el fin de incluir el mayor número de trabajos que se encuentren en la frontera del conocimiento.

Análisis bibliométrico: con el fin de identificar cómo está organizada la comunidad científica y la literatura, los artículos seleccionados se sometieron a un análisis bibliométrico. Merigo y Yang(2017)definen labibliometría como elanálisis cuantitativo de las características bibliográficas de un cuerpo de literatura y sustentan que el propósito del análisis bibliométrico es mapear la producción literaria en un campo de estudio dado y determinar la estructura de ese campo. En este sentido el análisis bibliométrico consistió en identificar publicación por años, áreas temáticas de investigación y publicaciones por revistas. Igualmente a través del software VOSviewer se realizaron mapas de citación, palabras clave y cocitación de autores.

Análisis de contenidos: con base en el objetivo de este estudio, el análisis del contenido consistió en la identificación de enfoques teóricos y metodológicos y futuras líneas de investigación a través de las lecturas a profundidad de los artículos seleccionados de acuerdo a los criterios de búsqueda.

Por último, es preciso señalar que, aun cuando la base de datos utilizada para extraer la literatura es completa y de prestigio, los documentos revisados tan solo responden a los criterios de búsqueda aplicados; por tanto, existe la posibilidad de que al modificarlos los 
resultados sean diferentes a la información que aquí se presenta.

\section{Resultados}

\section{Análisis bibliométrico}

De acuerdo a la ecuación de búsqueda definida para descargar los artículos ("academic entrepreneurship" AND "academic spin-off" OR "university spin-off") con la coincidencia en título, resumen y palabras clave, el primer resultado de búsqueda encontró 97 documentos distinción de autores- que han sido publicados en la base de datos Scopus a partir de 2001 en todas las áreas de estudio.

Al filtrar la búsqueda de los artículos con la restricción de tiempo (de enero de 2014 a octubre 2019) se obtuvieron 57 documentos que fueron revisados para realizar el análisis bibliométrico del presente estudio. Específicamente se seleccionaron 50 artículos, 3 conferencias, 2 capítulos de libro y 2 en revisión por la editorial, publicados durante el período 2014-2019.

Con base en el análisis de búsqueda se visualiza que hay una limitada producción respecto al número de publicaciones que abordan el tema del emprendimiento académico y spin-off en el contexto universitario en conjunto, aunque la temática presenta una tendencia en ascenso (figura 1). En 2018 se publicaron 11 documentos relacionados con el tema y en octubre de 2019 -al momento de escribir este artículo- se tenían 13, por lo que pudiera superarse el número año por año; esta tendencia parece indicar un gran interés en la comunidad científica por el tema, pues no se observa un declive significativo en el número de publicaciones alrededor del mismo.

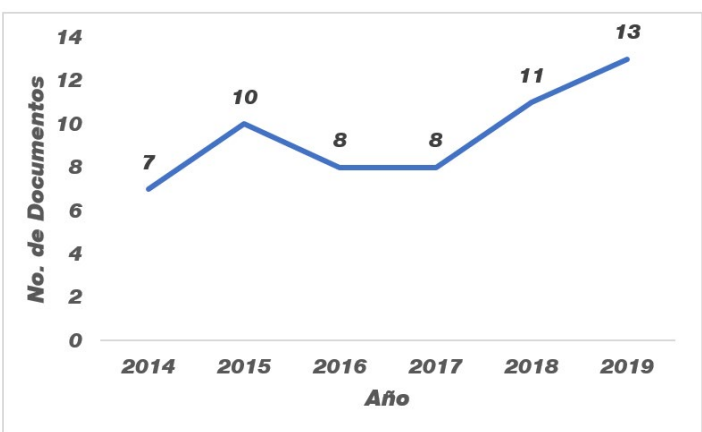

Figura 1. Número de documentos por año. Fuente: elaboración propia por parte de los autores.

Además, de acuerdo a la literatura, las áreas temáticas que más se han dirigido al estudio del emprendimiento académico y a la creación de spin-off son las de negocios y administración. Considerando los 97 documentos, que son el total de artículos de Scopus respecto al tópico aquí estudiado y que cumplen con la ecuación de búsqueda, se observa un dominio en las áreas de: negocios, administración y contabilidad (52,6 $\%$ ); ingeniería (17,9\%); economía, econometría y finanzas $(14,7 \%)$; el resto se distribuye tal como se muestra en la tabla 1.

Tabla 1. Número de publicaciones por área temática

\begin{tabular}{ccc}
\hline Área temática & $\begin{array}{c}\text { Número de } \\
\text { documentos }\end{array}$ & $\begin{array}{c}\text { Porcentaje } \\
\text { total (\%) }\end{array}$ \\
\hline $\begin{array}{c}\text { Negocios, } \\
\text { administración y } \\
\text { contabilidad }\end{array}$ & 50 & 52,6 \\
\hline Ingeniería & 17 & 17,9 \\
\hline $\begin{array}{c}\text { Economía, econometría } \\
\text { y finanzas }\end{array}$ & 14 & 14,7 \\
\hline Ciencias sociales & 9 & 9,5 \\
\hline Ciencias de la decisión & 4 & 4,2 \\
\hline $\begin{array}{c}\text { Ciencias de la } \\
\text { computación }\end{array}$ & 2 & 2,1 \\
\hline Artes y humanidades & 1 & 1,1 \\
\hline
\end{tabular}

Fuente: elaboración propia por parte de los autores. 
Este resultado apuntahacia el emprendimiento académico y spin-off universitario como un tópico multidisciplinario, lo que también se ve sustentado por la heterogeneidad de las revistas donde se publicaron los artículos en términos de su área de investigación. Como puede observarse en la figura 2 The Journal of Technology Transfer lidera con 14 publicaciones, seguida por: Journal of International Entrepreneurship con 5; Small Business Economics con 4; Journal of Industrial and Business Economics (Economia e Politica Industriale) con 2; Industry and Higher Education con 2 y Research Policy con 2.

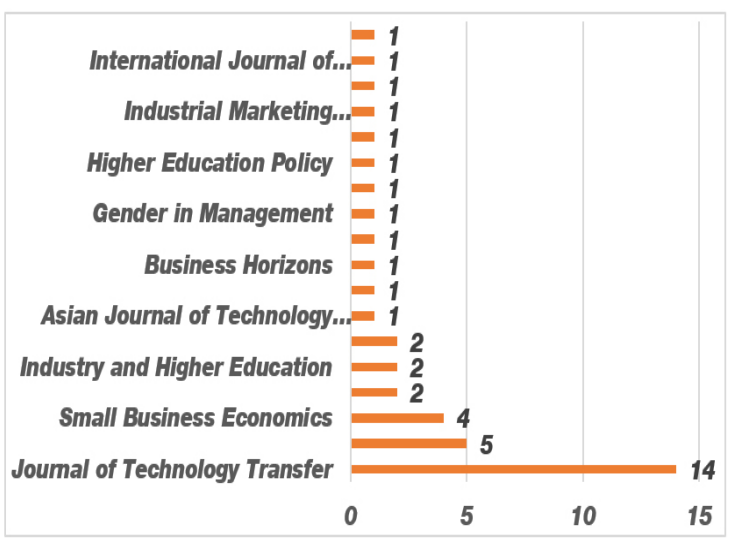

Figura 2. Número de publicaciones por revista. Fuente: elaboración propia por parte de los autores.

En la misma dirección y como resultado del tratamiento de la información sobre citación, palabras clave y coautoría a través del software VOSviewer, la figura 3 muestra las palabras con mayor número de coincidencias en los documentos analizados: emprendimiento académico; spin-off universitario; spin-off académico y transferencia de tecnología. Esta última representa el objetivo principal de la creación de empresas basadas en el conocimiento; es decir spin-off académicos generados a partir de resultados de proyectos de investigación.

De igual manera la variable educación es considerada una pieza clave en el tópico de emprendimiento, ya que se relaciona con desarrollar cultura y capacidades emprendedoras; además, en el emprendimiento académico la educación es proyectada en conocimiento puesto que genera el valor agregado de las empresas de base tecnológica y spin-off. 


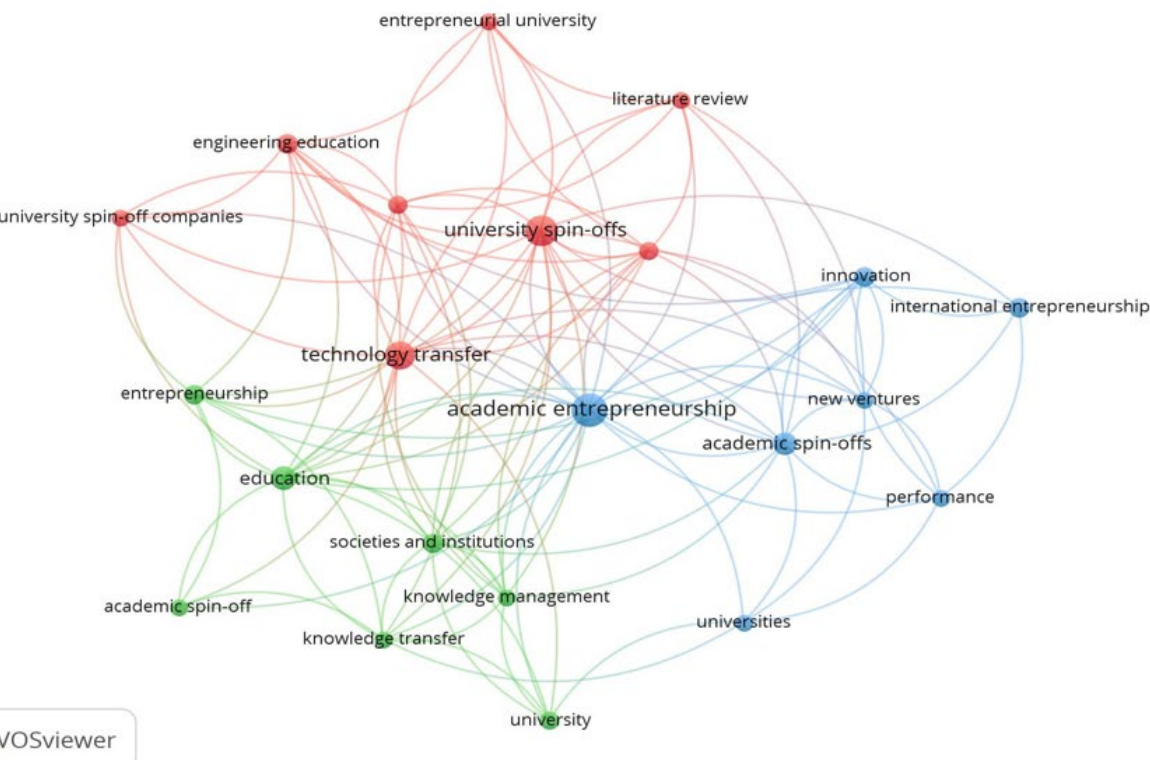

Figura 3. Palabras clave en artículos revisados. Fuente: elaboración propia por parte de los autores.

A la vez se identificó que el artículo más citado es "The influence of university departments on the evolution of entrepreneurial competencies in spin-off ventures" de Rasmussen, Mosey y Wright (2014) con 96 citas, seguido por: "“'To have and have not": founders' human capital and university start-up survival" de Criaco et al. (2014) con 49 citas y "The transformation of network ties to develop entrepreneurial competencies for university spin-offs" de Rasmussen, Mosey y Wright (2015) con 48 citas. La figura 4 muestra el mapa de red de citas por documento en función del número de documentos por autor y año de publicación en la base de datos; las burbujas del mismo color reflejan un mayor número de citas del documento de los autores y las líneas indican que hay una relación entre las citas de los autores (es decir, se citan entre sí).

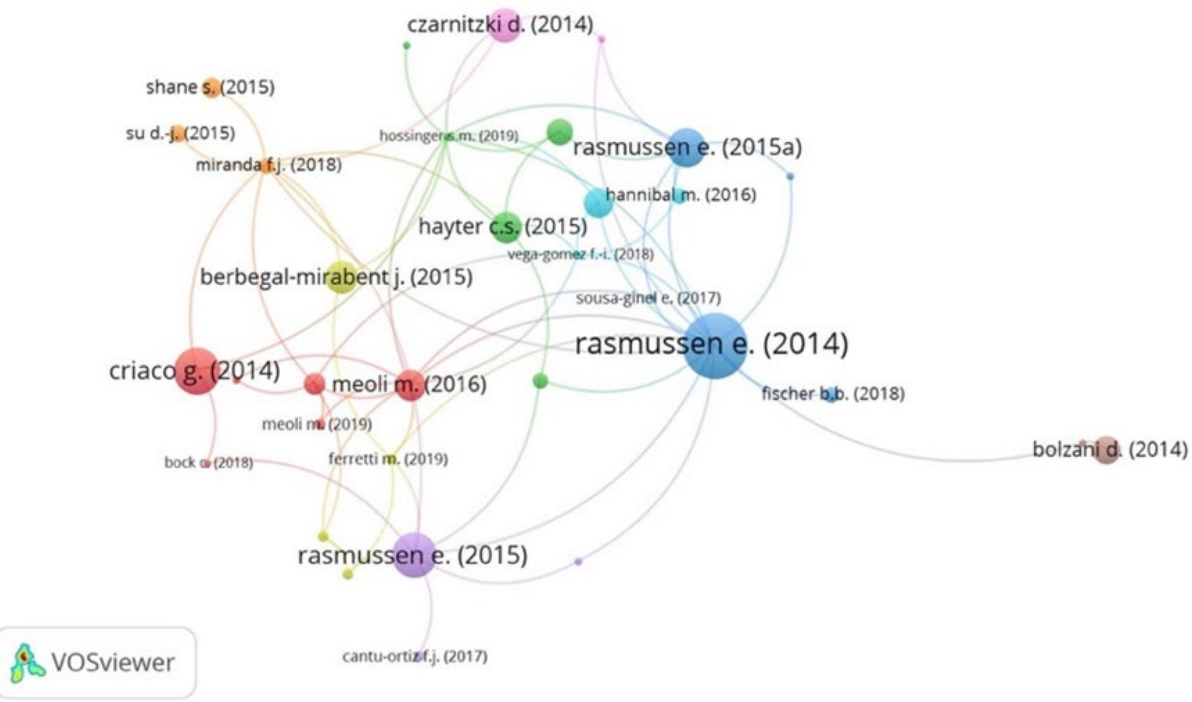

Figura 4. Análisis de citación por documento. Fuente: elaboración propia por parte de los autores. 
El análisis de cocitación por autores permite distinguir aquellos referentes que pueden proporcionar una visión de la disciplina o área del conocimiento. El mapa de red de cocitación (figura 5) muestra que Mike Wright es el autor con mayor número de referencias en el tópico de emprendimiento académico. Las líneas representan las conexiones que existen entre los autores y, al igual que la figura 4, las burbujas del mismo color una mayor correlación entre las citas del autor.

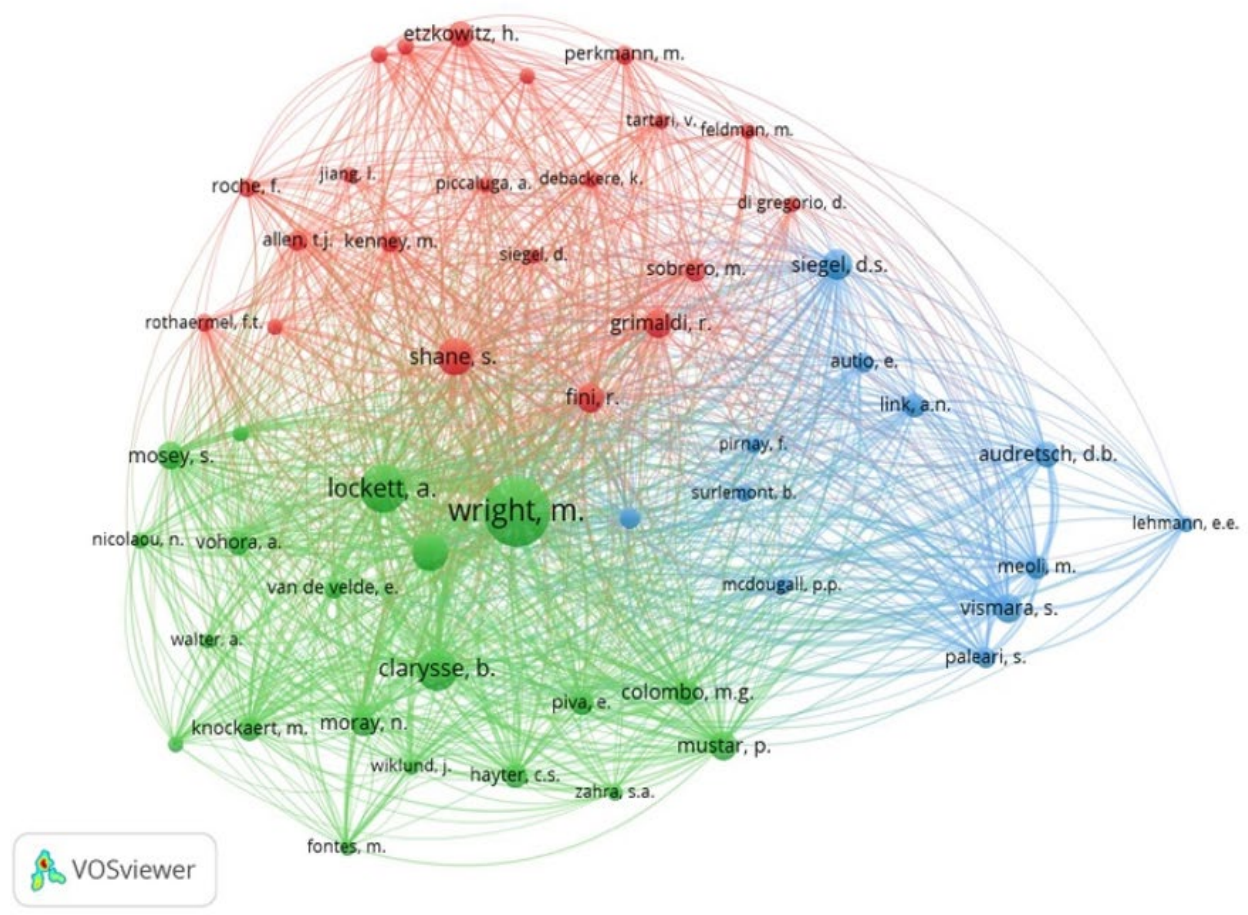

Figura 5. Cocitación de autores. Fuente: elaboración propia por parte de los autores.

\section{Análisis de contenido}

\section{Enfoques teóricos y metodológicos}

Se llevó a cabo una revisión detallada de los artículos para identificar marcos teóricos y metodológicos que han sido utilizados para estudiar el emprendimiento académico $\mathrm{y}$, en especial, la creación de empresas spin-off en el contexto universitario. En lo que refiere a los enfoques teóricos se encuentra una falta de homogeneidad del emprendimiento académico como objeto de estudio. Dicho de otra manera, hay diversidad de fenómenos y conceptos conformados por distintos recursos y capacidades de los agentes de un ecosistema de emprendimiento en el que se gesta la actividad empresarial; particularmente los spin-off generados en el entorno universitario conllevan a la participación de factores institucionales, organizacionales, colectivos e individuales. Esto ocasiona que haya enfoques teóricos heterogéneos en los estudios realizados hasta el momento.

Respecto a los 57 documentos revisados, con la idea de contribuir a homogeneizar la literatura, se percibe que los enfoques teóricos pueden ser clasificados en dos grupos: (i) aquellos que 
desarrollan marcos que refieren a un ecosistema emprendedor universitario (aspectos económico, social, político y cultural); (ii) otros que refieren al proceso de creación, desarrollo y desempeño de los spin-off. Así pues, la tabla 2 detalla los enfoques y las perspectivas de los diez artículos más citados: los estudios de Rasmussen, Mosey y Wright (2014) y Rasmussen y Wright (2015) consideran como marco teórico el entorno universitario y las competencias empresariales; por su parte Berbegal-Mirabent, Ribeiro-Soriano y Sánchez García (2015) desarrollan un marco teórico relacionado con programas de apoyo, marco normativo y recursos (capital humano e infraestructura).

Tabla 2. Enfoques teóricos en los artículos más citados

\begin{tabular}{|c|c|c|c|c|}
\hline Enfoque teórico & Perspectiva & Autor & Título & $\begin{array}{l}\text { Número } \\
\text { de citas }\end{array}$ \\
\hline \multirow[t]{6}{*}{$\begin{array}{l}\text { Ecosistema de } \\
\text { emprendimiento } \\
\text { universitario }\end{array}$} & $\begin{array}{l}\text { Competencias } \\
\text { empresariales } \\
\text { Evolución de competencias }\end{array}$ & $\begin{array}{l}\text { Rasmussen, Mosey y } \\
\text { Wright (2014) }\end{array}$ & $\begin{array}{l}\text { "The influence of university } \\
\text { departments on the } \\
\text { evolution of entrepreneurial } \\
\text { competencies in spin-off } \\
\text { ventures" }\end{array}$ & 96 \\
\hline & $\begin{array}{l}\text { Emprendimiento académico } \\
\text { Capital humano fundador }\end{array}$ & Criaco et al. (2014) & $\begin{array}{l}\text { ““To have and have not”: } \\
\text { Founders' human capital } \\
\text { and university start-up } \\
\text { survival” }\end{array}$ & 49 \\
\hline & $\begin{array}{l}\text { Competencias } \\
\text { empresariales } \\
\text { Recursos para creación de } \\
\text { competencias }\end{array}$ & $\begin{array}{l}\text { Rasmussen y Wright } \\
\text { (2015) }\end{array}$ & $\begin{array}{l}\text { "How can universities } \\
\text { facilitate academic spin- } \\
\text { offs? An entrepreneurial } \\
\text { competency perspective" }\end{array}$ & 48 \\
\hline & $\begin{array}{l}\text { Redes } \\
\text { Competencias } \\
\text { empresariales }\end{array}$ & $\begin{array}{l}\text { Rasmussen, Mosey y } \\
\text { Wright (2015) }\end{array}$ & $\begin{array}{l}\text { "The transformation } \\
\text { of network ties to } \\
\text { develop entrepreneurial } \\
\text { competencies for university } \\
\text { spin-offs" }\end{array}$ & 37 \\
\hline & $\begin{array}{l}\text { Programas de apoyo } \\
\text { Recursos: capital humano, } \\
\text { infraestructura } \\
\text { Marco normativo }\end{array}$ & $\begin{array}{l}\text { Berbegal-Mirabent, } \\
\text { Ribeiro-Soriano y } \\
\text { Sánchez García (2015) }\end{array}$ & $\begin{array}{l}\text { "Can a magic recipe } \\
\text { foster university spin-off } \\
\text { creation?" }\end{array}$ & 27 \\
\hline & $\begin{array}{l}\text { Emprendimiento académico } \\
\text { (emprendimiento público) } \\
\text { Motivación } \\
\text { Emprendedor académico }\end{array}$ & Hayter (2015) & $\begin{array}{l}\text { "Public or private } \\
\text { entrepreneurship? } \\
\text { Revisiting motivations } \\
\text { and definitions of } \\
\text { success among academic } \\
\text { entrepreneurs" }\end{array}$ & 24 \\
\hline
\end{tabular}




\begin{tabular}{|c|c|c|c|c|}
\hline Enfoque teórico & Perspectiva & Autor & Título & $\begin{array}{l}\text { Número } \\
\text { de citas }\end{array}$ \\
\hline \multirow{6}{*}{$\begin{array}{l}\text { Creación, desarrollo y } \\
\text { desempeño de spin-off } \\
\text { universitarios }\end{array}$} & Costo social & $\begin{array}{l}\text { Czarnitzki, Rammer y } \\
\text { Toole (2014) }\end{array}$ & $\begin{array}{l}\text { "University spin-offs } \\
\text { and the "performance }\end{array}$ & 28 \\
\hline & Desempeño & & premium & \\
\hline & $\begin{array}{l}\text { Apoyo administrativo } \\
\text { universitario }\end{array}$ & Meoli y Vismara (2016) & $\begin{array}{l}\text { "University support and the } \\
\text { creation of technology and } \\
\text { non-technology academic } \\
\text { spin-offs" }\end{array}$ & 21 \\
\hline & $\begin{array}{l}\text { Política universitaria para } \\
\text { creación de spin-off } \\
\text { Incentivos monetarios }\end{array}$ & $\begin{array}{l}\text { Muscio, Quaglione y } \\
\text { Ramaciotti (2016) }\end{array}$ & $\begin{array}{l}\text { "The effects of university } \\
\text { rules on spinoff creation: } \\
\text { The case of academia in } \\
\text { Italy" }\end{array}$ & 25 \\
\hline & Riesgo empresarial & & & \\
\hline & $\begin{array}{l}\text { Contexto macroeconómico } \\
\text { Desempeño de spin-off }\end{array}$ & Bolzani et al. (2014) & $\begin{array}{l}\text { "University spin-offs and } \\
\text { their impact: Longitudinal } \\
\text { evidence from Italy" }\end{array}$ & 18 \\
\hline
\end{tabular}

Fuente: elaboración propia por parte de los autores.

En cuanto a los métodos de investigación utilizados en estudios relacionados con el emprendimiento académico y la creación de spin-off, la mayoría de los trabajos son empíricos. Dentro de los trabajos empíricos destacan los estudios de casos con enfoques cualitativos. No se encontró una sola investigación que utilizara alguna mezcla de datos cualitativos y cuantitativos del mismo conjunto de individuos, empresas, industrias y/o países.

Miranda, Chamorro-Mera y Rubio (2017) enfatizan que entre los trabajos cuantitativos las técnicas más utilizadas son los modelos de regresión lineal múltiple, seguidos de los modelos no lineales (tales como logit, probit o regresión logística) y los modelos de ecuaciones estructurales. Además, los autores señalan que algunos trabajos analizados se limitan a estadística descriptiva para presentar sus hallazgos. De los artículos obtenidos en la base de datos, la tabla 3 presenta los diez estudios con el mayor número de citas y el enfoque metodológico de cada uno de ellos; puede apreciarse que solo cuatro son de tipo cualitativo y seis cuantitativo, los cuales utilizan técnicas estadísticas y econométricas. 
Tabla 3. Metodología en los artículos más citados

\begin{tabular}{|c|c|c|c|c|}
\hline Autor & Título & Tipo & Metodología & \\
\hline $\begin{array}{l}\text { Rasmussen, Mosey y } \\
\text { Wright (2014) }\end{array}$ & $\begin{array}{l}\text { "The influence of university } \\
\text { departments on the evolution of } \\
\text { entrepreneurial competencies in } \\
\text { spin-off ventures" }\end{array}$ & Empírico & Estudio de caso/entrevistas & \multirow{4}{*}{ Cualitativo } \\
\hline $\begin{array}{l}\text { Rasmussen y Wright } \\
(2015)\end{array}$ & $\begin{array}{l}\text { "How can universities facilitate } \\
\text { academic spin-offs? An } \\
\text { entrepreneurial competency } \\
\text { perspective” }\end{array}$ & Teórico & Revisión documental & \\
\hline $\begin{array}{l}\text { Rasmussen, Mosey y } \\
\text { Wright (2015) }\end{array}$ & $\begin{array}{l}\text { "The transformation of network } \\
\text { ties to develop entrepreneurial } \\
\text { competencies for university spin- } \\
\text { offs" }\end{array}$ & Empírico & Estudio de caso/entrevistas & \\
\hline Hayter (2015) & $\begin{array}{l}\text { “Public or private } \\
\text { entrepreneurship? Revisiting } \\
\text { motivations and definitions } \\
\text { of success among academic } \\
\text { entrepreneurs" }\end{array}$ & Empírico & Entrevistas & \\
\hline
\end{tabular}

\begin{tabular}{|c|c|c|c|c|}
\hline Criaco et al. (2014) & $\begin{array}{l}\text { ““To have and have not”: } \\
\text { Founders' human capital and } \\
\text { university start-up survival” }\end{array}$ & Empírico & Regresión logística & \multirow{6}{*}{ Cuantitativo } \\
\hline $\begin{array}{l}\text { Czarnitzki, Rammer y } \\
\text { Toole (2014) }\end{array}$ & $\begin{array}{l}\text { “University spin-offs and the } \\
\text { "performance premium"” }\end{array}$ & Empírico & Modelos de crecimiento & \\
\hline $\begin{array}{l}\text { Berbegal-Mirabent, } \\
\text { Ribeiro-Soriano y } \\
\text { Sánchez García (2015) }\end{array}$ & $\begin{array}{l}\text { “Can a magic recipe foster } \\
\text { university spin-off creation?" }\end{array}$ & Empírico & $\begin{array}{l}\text { Análisis comparativo } \\
\text { cualitativo/crisp set y fuzzy } \\
\text { set }\end{array}$ & \\
\hline Meoli y Vismara (2016) & $\begin{array}{l}\text { "University support and the } \\
\text { creation of technology and non- } \\
\text { technology academic spin-offs" }\end{array}$ & Empírico & Regresión binomial & \\
\hline $\begin{array}{l}\text { Muscio, Quaglione y } \\
\text { Ramaciotti (2016) }\end{array}$ & $\begin{array}{l}\text { "The effects of university rules } \\
\text { on spinoff creation: The case of } \\
\text { academia in Italy" }\end{array}$ & Empírico & Modelo econométrico & \\
\hline Bolzani et al. (2014) & $\begin{array}{l}\text { "University spin-offs and their } \\
\text { impact: Longitudinal evidence } \\
\text { from Italy" }\end{array}$ & Empírico & stadística descriptiva & \\
\hline
\end{tabular}

Fuente: elaboración propia por parte de los autores.

Se identificó que los artículos de Rasmussen, Mosey y Wright $(2014,2015)$ y Rasmussen y Wright (2015) mantienen un enfoque cualitativo, realizan estudios de caso y la triangulación de datos la incorporan a través de fuentes documentales y entrevistas. De igual manera Hayter (2015) utiliza la entrevista en su estudio como principal técnica para obtener información.

También se observa que en los últimos años el número de trabajos cuantitativos ha aumentado, lo que Miranda, Chamorro y Rubio (2018) interpretan como una maduración de la disciplina del emprendimiento que ha pasado de la etapa inicial de desarrollo de teorías a la etapa de validación. Hossinger, Chen y Werner (2019) señalan que una posible explicación para esta situación puede ser que en las primeras etapas de la investigación académica sobre emprendimiento los académicos carecían de datos confiables, así como de teorías y marcos para realizar análisis cuantitativos; por tanto, el método cualitativo fue un medio más efectivo para describir fenómenos y explorar factores influyentes. 


\section{Futuras líneas de investigación}

A pesar de la creciente literatura en emprendimiento académico y spin-off en el contexto universitario, que indica el interés por parte de la comunidad científica, la literatura aún está fragmentada y subteorizada (Rothaermel, Agung and Jiang, 2007; Schmitz et al., 2016; Guerrero, Cunningham and Urbano, 2017; Clauss, Moussa and Kesting, 2018; Mathisen and Rasmussen, 2019); de ahí que se requieran estudios más sistemáticos y holísticos (Hayter, 2015; Wright, Siegel and Mustar, 2017; Bae, 2018), considerando los aspectos económicos y sociales del emprendimiento académico y las

Tabla 4. Futuras líneas de investigación diferentes etapas de los spin-off universitarios (creación, funcionamiento y desempeño) (Bolzani et al., 2014; Martins and Polanski, 2015; Huynh et al., 2017; Bock et al., 2018; Civera and Meoli, 2018; Fischer et al., 2018; Bae, 2018; Hossinger, Chen and Werner, 2019; Civera, Meoli and Vismara, 2019), que en diversos contextos pueden desarrollar estudios empíricos relacionados con diferentes modelos de negocios generados en las universidades para comercializar sus proyectos de investigación (Cantu-Ortiz et al., 2017; Jung and Kim, 2018). En la tabla 4 se pueden observar las futuras líneas y brechas de investigación que se pueden derivar de algunos de los artículos consultados.

\section{Futuras líneas de investigación}

Considerar análisis longitudinales relacionados con el proceso de creación de spin-off académicos dado que el proceso de emprendimiento es largo, complejo y participan diversos niveles en su gestión. El capital humano y social de los académicos, los estilos cognitivos y las capacidades evolucionan con el tiempo durante el proceso del spin-off

Adoptar una perspectiva más integrada, prestando atención a la interacción entre los diferentes predictores en varios niveles (es decir, el individuo, la empresa, la organización y el macroambiente) para que se pueda encontrar una combinación óptima

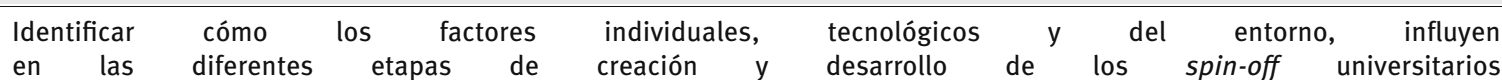

Analizar modelos de negocios de spin-off universitarios que han sido exitosos

Estudiar datos a nivel individual para comprender las características internas y los microprocesos del emprendimiento en el entorno universitario

El cómo los actores individuales tanto académicos como graduados responden y evolucionan en relación con diferentes contextos organizacionales, procesos heterogéneos de creación de conocimiento y capacidades emprendedoras

Diseñar estrategias para incentivar el emprendimiento académico

Tipos y mecanismos de modelos de triple hélice en diversas regiones para el fomento del vínculo universidad-industria

Evaluar el desempeño de spin-off académicos ya establecidos

Estudiar las motivaciones que conducen a la creación de spin-off académicos; particularmente las motivaciones de los estudiantes de doctorado que los lleva a ser emprendedores

Explorar el impacto de la regulación universitaria (a nivel departamental o individual) en el emprendimiento académico

Identificar el cómo la generación de spin-off influye en el desempeño universitario, en las actividades de investigación y en la capacidad de la universidad para atraer contratos de investigación

Estudiar la interacción entre las motivaciones de los emprendedores académicos, las políticas y los programas de apoyo al emprendimiento académico

Investigar y conceptualizar diferentes “modelos de negocios” para los spin-off universitarios

Profundizar el estudio de las motivaciones y definiciones de éxito entre los empresarios académicos, cómo evolucionan y por qué 
Evaluar cómo las universidades pueden ayudar a las empresas de base tecnológica de alto crecimiento según los tipos de nuevas empresas, el tipo de universidades y los contextos en los que operan. Investigaciones que integren la perspectiva de la empresa y la universidad

El cómo las universidades pueden manejar y fomentar las relaciones con los actores externos que brindan información crucial para el desarrollo de competencias en los spin-off

Realizar estudios de caso para comprender mejor lo que fomenta las spin-off universitarias. Del mismo modo otros métodos de calibración de datos pueden proporcionar información adicional. Combinar diversos métodos cuantitativos con el método de análisis cualitativo comparativo (QCA, por sus siglas en inglés)

Fuente: elaboración propia por parte de los autores.

\section{Conclusión}

Con el presente trabajo se ha explorado y descrito cómo está organizada la literatura sobre emprendimiento académico y spin-off universitarios. Posteriormente se han identificado enfoques teóricos, metodologías y brechas que podrían conducir a futuros trabajos de investigación. Para ello se utilizó la base de datos Scopus donde se filtró la búsqueda de textos desde enero de 2014 hasta octubre de 2019, por considerarlos que están en la frontera del conocimiento. La muestra final analizada consistió en un total de 57 artículos.

Un análisis bibliométrico mostró una limitada producción respecto al número de publicaciones del tema de emprendimiento académico y creación de spin-off, pero con tendencia ascendente. Esto indica el interés de la comunidad científica en el tema. Las revistas y las áreas de investigación revelaron que el tema está siendo estudiado por varias disciplinas y áreas donde prevalecen los estudios de negocios, administración e ingeniería. El análisis de palabras clave arrojó que los estudios de emprendimiento y spin-off en el contexto universitario son considerados como el medio para transferir tecnología del entorno científico al empresarial.

El análisis de contenido de los artículos revisados presentó que a pesar de la creciente literatura, esta no es homogénea en términos de los fenómenos y conceptos que aborda; pues están conformados por distintos recursos y capacidades de los agentes de un ecosistema de emprendimiento. Esto implica que lo estudios de emprendimiento académico y creación de spinoff consideran procesos en múltiples niveles $\mathrm{y}$ diversos contextos. Como resultado de lo anterior, la literatura alrededor del tema se encuentra fragmentada y con poca fundamentación teórica.

Enloquerespectaalosenfoquesmetodológicos los trabajos cuantitativos se han incrementado, utilizando técnicas estadísticas y econométricas. Las brechas de investigación encontradas señalan la necesidad de estudios futuros relacionados con aspectos económicos y sociales y factores institucionales, organizacionales e individuales. Además, la investigación que logre incorporar una mezcla de enfoques cualitativos y cuantitativos puede ofrecer conocimientos complementarios.

Finalmente es preciso reiterar que este estudio solo representa un acercamiento al área de investigación de emprendimiento académico y spin-off universitarios, ya que solo se han considerado los documentos que resultaron de los criterios de búsqueda definidos en una base de datos específica.

\section{Referencias}

Audretsch, D. (2014). From the entrepreneurial university to the university for the entrepreneurial society. The Journal of Technology Transfer, 39(3), 313-321.

Autio, E. et al. (2014). Entrepreneurial Innovation: The importance of context. Research Policy, 43(7), 1097-1108.

Bae, Z.-T. (2018). Academic entrepreneurship: Commercialization of university research and entrepreneurship education at an entrepreneurial 
university. En Portland International Conference on Management of Engineering and Technology: Managing Technological Entrepreneurship: The Engine for Economic Growth, Proceedings, Honolulu, Hawaii, USA.

Berbegal-Mirabent, J., Ribeiro-Soriano, D. and Sánchez García, J. (2015). Can a magic recipe foster university spin-off creation? Journal of Business Research, 68(11), 2272-2278.

Bock, C. et al. (2018). Are public financing schemes beneficial for university spin-offs and the technology transfer of innovations? International Journal of Innovation Management, 22(6), 1-30.

Boh, W., De-Haan, U. and Strom, R. (2016). University Technology Transfer Through Entrepreneurship: Faculty and Students in Spinoffs. The Journal of Technology Transfer, 41(4), 661-669.

Bolzani, D. et al. (2014). University spin-offs and their impact: Longitudinal evidence from Italy. Journal of Industrial and Business Economics, 41(4), 237-263.

Bradley, S., Hayter, C. and Link, A. (2013). Models and methods of university technology transfer. Foundations and Trends in Entrepreneurship, 9(6), 571-650.

Carayannis, E. (2017). Series Foreword. En Peris-Ortiz, M. (Ed.), Entrepreneurial Universities exploring the academic and innovative dimensions of entrepreneurship in higher education (pp. v-vii). Washington, USA: Springer.

Cantu-Ortiz, F. et al. (2017). Spreading academic entrepreneurship: Made in Mexico. Business Horizons, 60(4), 541-550.

Civera, A. and Meoli, M. (2018). Does university prestige foster the initial growth of academic spin-offs? Economia e Politica Industriale, 45(2), 1-32.

Civera, A., Meoli, M. and Vismara, S. (2019). Do academic spinoffs internationalize? The Journal of Technology Transfer, 44(2), 381-403.

Clauss, T., Moussa, A. and Kesting, T. (2018). Entrepreneurial university: A stakeholder-based conceptualisation of the current state and an agenda for future research. International Journal of Technology Management, 77(1/2/3), 109-144.

Clarysse, B., Tartari, V. and Salter, A. (2011). The impact of entrepreneurial capacity, experience and organizational support on academic entrepreneurship. Research Policy, 40(8), 10841093.

Criaco, G. (2014). "To have and have not": founders' human capital and university start-up survival. The Journal of Technology Transfer, 39(4), 567-593.

Cunningham, J. and Link, A. (2015). Fostering universityindustry R\&D collaborations in European Union countries. International Entrepreneurship and Management Journal, 11(4), 849-860.

Cunningham, J. and O'Reilly, P. (2018). Macro, meso and micro perspectives of technology transfer. The Journal of Technology Transfer, 43(3), 545-557.

Czarnitzki, D., Rammer C. and Toole, A. (2014). University spin-offs and the "performance premium." Small Business Economics, 43(2), 309-326.

Etzkowitz, H. (2017). Innovation lodestar: The entrepreneurial university in a stellar knowledge firmament. Technological Forecasting \& Social Change, 123, 122-129.

Etzkowitz, H. et al. (2000). The future of the university and the university of the future: Evolution of ivory tower to entrepreneurial paradigm. Research Policy, 29(2), 313-330.

Fischer, B. et al. (2018). Quality comes first: universityindustry collaboration as a source of academic entrepreneurship in a developing country. The Journal of Technology Transfer, 43(2), 263-284.

Guerrero, M. and Urbano, D. (2012). The development of an entrepreneurial university. The Journal of Technology Transfer, 37(1), 43-74.

Guerrero, M., Cunningham, J. and Urbano, D. (2015). Economic impact of entrepreneurial universities' activities: An exploratory study of the United Kingdom. Research Policy, 44(3), 748-764.

Guerrero, M., Urbano, D. and Gajón, E. (2017). Higher education entrepreneurial ecosystems: Exploring the role of business incubators in an emerging economy. International Review of Entrepreneurship, 15(2), 01-28.

Hayter, C. (2015). Public or private entrepreneurship? Revisiting motivations and definitions of success among academic entrepreneurs. The Journal of Technology Transfer, 40(6), 1003-1015.

Hayter, C. et al. (2018). Conceptualizing academic entrepreneurship ecosystems: A review, analysis 
and extension of the literature. The Journal of Technology Transfer, 43(4), 1039-1082.

Hossinger, S., Chen, X. and Werner, A. (2019). Drivers, barriers and success factors of academic spin-offs: A systematic literature review. Management Review Quarterly, 70(1), 97-134.

Huynh, T. et al. (2017). University spin-off's performance: Capabilities and networks of founding teams at creation phase. Journal of Business Research Volume, 78, 10-22.

Jung, H. and Kim, B. (2018). Determinant factors of university spin-off: The case of Korea. The Journal of Technology Transfer, 43(4), 1631-1646.

Liñán, F. and Fayolle, A. (2015). A systematic literature review on entrepreneurial intentions: Citation, thematic analyses, and research agenda. International Entrepreneurship and Management Journal, 11(4), 907-933.

Lockett, A. and Wright, M. (2005). Resources, capabilities, risk capital and the creation of university spin-out companies. Research Policy, 34(7), 1043-1057.

Mathisen, M. and Rasmussen, E. (2019). The development, growth, and performance of university spin-offs: A critical review. The Journal of Technology Transfer, 44(6), 1891-1938.

Martins, P. and Plonski, G. (2015). Science spin-offs in the context of Brazilian Academic entrepreneurship. En IAMOT 2015 - 24th International Association for Management of Technology Conference: Technology, Innovation and Management for Sustainable Growth, Proceedings, Cape Town, South Africa.

Mascarenhas, C. et al. (2017). Entrepreneurial university: Towards a better understanding of past trends and future directions. Journal of Enterprising Communities: People and Places of Global Economy, 11(3), 316-338.

Meoli, M. and Vismara, S. (2016). University support and the creation of technology and non-technology academic spin-offs. Small Business Economics, 47(2), 345-362.

Merigo, J. and Yang, J. (2017). A bibliometric analysis of operations research and management science. Omega. International Journal of Management Science, 73, 37-48.
Miranda, F., Chamorro-Mera, A. and Rubio, S. (2017). Academic entrepreneurship in Spanish universities: An analysis of the determinants of entrepreneurial intention. European Research on Management and Business Economics, 23(2), 113-122.

Miranda, F., Chamorro, A. and Rubio, S. (2018). Rethinking university spin-off: A critical literature review and a research agenda. The Journal of Technology Transfer, 43(4), 1007-1038.

Mosey, S., Guerrero, M. and Greenman, A. (2016). Technology entrepreneurship research opportunities: Insights from across Europe. The Journal of Technology Transfer, 42(1), 1-9.

Muscio, A., Quaglione, D. and Ramaciotti, L. (2016). The effects of university rules on spinoff creation: The case of academia in Italy. Research Policy, 45(7), 1386-1396.

O'Shea, R., Chugh, H. and Allen, T. (2008). Determinants and consequences of university spin-off activity: A conceptual framework. The Journal of Technology Transfer, 33(6), 653-666.

O'Shea, R. et al. (2014). University-based entrepreneurship: A synthesis of the literature. En O'Shea, R. and Allen, T. (Ed.), Building Technology Transfer Within Research Universities (pp. 33-59). Massachusetts, USA: MIT Press.

Rao, B. and Mulloth, B. (2017). The role of universities in encouraging growth of technology-based new ventures. International Journal of Innovation and Technology Management, 14(4), 1-22.

Rasmussen, E., Mosey, S. and Wright, M. (2014). The influence of university departments on the evolution of entrepreneurial competencies in spinoff ventures. Research Policy, 43(1), 92-106.

Rasmussen, E., Mosey, S. and Wright, M. (2015). The transformation of network ties to develop entrepreneurial competencies for university spin-offs. Entrepreneurship \& Regional Development, 27(7-8), 430-457.

Rasmussen, E. and Wright, M. (2015). How can universities facilitate academic spin-offs? An entrepreneurial competency perspective. The Journal of Technology Transfer, 40(5), 782-799.

Rothaermel, F., Agung, S. and Jiang, L. (2007). University entrepreneurship: A taxonomy of the literature. Industrial and Corporate Change, 16(4), 691-791. 
Schmitz, A. et al. (2016). Innovation and entrepreneurship in the academic setting: A systematic literature review. International Entrepreneurship and Management Journal, 13(2), 369-395.

Siegel, D. (2018). Academic Entrepreneurship: Lessons Learned for Technology Transfer Personnel and University Administrators. En Libaers, D. (Ed.), Volume 1: University Technology Transfer and Academic Entrepreneurship (pp. 1-21). Pulau Ujong, Singapore: World Scientific Publishing Company.

Urban, B. and Chantson, J. (2017). Academic entrepreneurship in South Africa: Testing for entrepreneurial intentions. The Journal of Technology Transfer, 44(2), 948-980.

Wright, M., Siegel, D. and Mustar, P. (2017). An emerging ecosystem for student start-ups. The Journal of Technology Transfer, 42(4), 909-922. 